\title{
Entrevista a Alejandra Borrero, María Victoria Estrada y León David Cobo, directivos del equipo pedagógico y artístico de Casa E Social a cargo del Proyecto VICTUS
}

\author{
Claudia Victoria Girón Ortiz/ Redunipaz - Red universitaria por la paz
}

Gabriel Izquierdo

\section{Introducción}

En el marco de los Acuerdos de Paz desarrollados a partir de la búsqueda de una solución política negociada entre el gobierno del ex Presidente Juan Manuel Santos y la guerrilla de las FARC - que comenzó con los diálogos de La Habana (Cuba) en el año 2012 - se planteó la necesidad de crear un sistema integral de mecanismos judiciales y extra judiciales con el fin de lograr la satisfacción de los derechos de todas las víctimas del conflicto armado, la aceptación de responsabilidad por parte de los victimarios, y la seguridad jurídica de quienes se sometiesen a las reglas del juego con el fin de facilitar la transición del conflicto violento hacia una paz estable y duradera.

Estos mecanismos judiciales y extrajudiciales articulados en el denominado "Sistema Integral de Verdad, Justicia, Reparación y No Repetición" (SIVJRNR) operan a través de las siguientes instituciones: La Comisión para el Esclarecimiento de la Verdad, la Convivencia y la No Repetición (Comisión de la Verdad-CEV); la Unidad para la Búsqueda de Personas dadas por Desaparecidas en el Contexto y en razón del Conflicto Armado; la Jurisdicción Especial para la Paz (JEP); y las Medidas de Reparación Integral.

En los últimos tres años se ha hecho cada vez más evidente que la postura crítica por parte de los sectores políticos más representativos de quienes se oponen a los avances en la implementación de los Acuerdos de Paz, encabezados por el Centro Democrático (actual partido de gobierno) no expresa la intención de aportar constructivamente al fortalecimiento y la cualificación del SIVJRNR en aras del bien común, sino más bien, es la expresión de una fuerte animadversión, no solamente contra el proceso de paz que fuera liderado por el ex Presidente Juan Manuel Santos, sino contra la guerrilla de las FARC, construida política, social y mediáticamente durante más de cinco décadas, como "el único, el peor y el más grande enemigo interno de la nación." En el caso colombiano, es importante señalar, que es aunque es innegable que la guerrilla de las FARC cometió crímenes de Lesa Humanidad y crímenes de guerra, debe quedar absolutamente claro para la sociedad que este grupo armado ilegal no fue el único actor que incurrió en este tipo de prácticas atroces que atentaron contra la vida y la dignidad de millones de colombianos. Desde esta perspectiva, las FARC no son el único sector de los victimarios que debe someterse a confesar toda la verdad, reconocer sus responsabilidades frente a los daños causados, y pedir perdón a las víctimas y a la sociedad en su conjunto, comprometiéndose a no volver a repetir los actos de barbarie que cometieron en el pasado.

El triunfo del no en el plebiscito en octubre del año 2016, después de la firma de los Acuerdos de Paz en La Habana, fue el resultado de una campaña de desprestigio orquestada por los opositores políticos de Santos, vehiculada a través de un poderoso sector de los medios masivos de comunicación, y basada en información falsa sobre el contenido de dichos acuerdos. Desafortunadamente esa campaña de desprestigio contra los Acuerdos de Paz y contra el SIVJRNR, se ha venido ampliando y recrudeciendo desde mediados del año 2018, a partir de la elección de Iván Duque Márquez, Presidente de la República designado por el ex Presidente Álvaro Uribe Vélez, actual Senador y cabeza principal del Centro Democrático.

Teniendo en cuenta este panorama tan complejo, es claro que uno de los principales intereses del actual gobierno es debilitar el SIVJRNR, atacando las instituciones que lo conforman, cuestionando su legitimidad y reduciendo el presupuesto destinado para su funcionamiento (https://pacifista.tv/notas/jep-comision-de-la-verdad-2018-centro-democratico-ataques-reformas). Para diferentes analistas lo que realmente está en juego en el contexto transicional colombiano es el monopolio de la verdad, dado que para el sistema integral que se desprende de los Acuerdos de Paz, el eje fundamental de la transición hacia un escenario distinto al de la guerra permanente, es el establecimiento de un nuevo pacto social fundamentado en la verdad y en la justicia restaurativa, a partir del cual se construyan las garantías de no repetición de los hechos violentos. El carácter restaurativo de la justicia depende en buena parte del esclarecimiento de la verdad y de su sentido transformador, puesto que si la verdad puede contribuir al reconocimiento público de las víctimas como sujetos de derechos y testigos históricos, cuyos testimonios dan cuenta de los impactos -daños y pérdidas- individuales y colectivos ocasionados en el contexto del conflicto, y al reconocimiento de los diferentes niveles de responsabilidad 
de los actores - civiles y armados, legales e ilegales - que determinaron $\mathrm{y} / \mathrm{o}$ perpetraron acciones violentas que atentaron contra la vida y la dignidad, esa rendición de cuentas por parte de los victimarios podría constituir un primer paso para restaurar la dignidad de las víctimas, los vínculos intersubjetivos y las relaciones humanas al interior de una sociedad tan herida como la nuestra.

En consonancia con la perspectiva crítica de Alejandro Castillejo, considero que, si bien, todo escenario transicional plantea horizontes y posibilidades para avanzar hacia un futuro diferente, las esperanzas de lograr las transformaciones que la sociedad colombiana requiere para salir del conflicto violento no dependen exclusivamente de los engranajes de la institucionalidad encargada de implementar el SIVJRNR, sino, principalmente, de los escenarios locales y regionales donde se están generando nuevas dinámicas relacionales de cercanía y projimidad con los otros, aún en medio del caos y la conflictividad reinantes (Castillejo 2018; https:/lapipa. co/la-paz-desde-lo-pequeno-alejandro-castillejo). Son precisamente esas experiencias de cercanía y projimidad con los otros, las que posibilitan un cambio de paradigma que implica de-construir la noción del otro como "enemigo"; es decir, de-construir esa "otredad negativa" (Feierstein, 2007) que durante décadas ha alimentado el odio y la polarización entre los colombianos, impidiéndoles reconocer al adversario o contradictor político como un semejante, como un ser humano con virtudes y defectos y como un interlocutor válido). ${ }^{1}$

Dentro de esos esfuerzos a pequeña escala por construir escenarios de convivencia pacífica, a partir del reconocimiento y el respeto de la alteridad y de la diversidad, es importante destacar el papel que a lo largo de la historia han jugado los sectores artísticos, educativos y culturales. Uno de los procesos artísticos más interesantes que se ha venido desarrollando en los últimos años en Colombia es el Proyecto VICTUS, cuyo nombre significa "víctimas victoriosas" y apela al sentido de la resiliencia como motor de la superación personal de aquellos protagonistas del conflicto armado que han logrado reconstruir sus proyectos de vida, constituyéndose en un ejemplo vivo de esperanza para la sociedad.

VICTUS se articula como un laboratorio de memoria y reconciliación entre víctimas civiles y desmovilizados de los diferentes grupos armados y está dirigido por un equipo interdisciplinario a cargo de la actriz y dramaturga Alejandra Borrero con sede en Casa E-un multiplex artístico ubicado en Bogotá- donde, de manera itinerante, se presentan obras de teatro, exposiciones, charlas y conversatorios; en buena parte relacionados con la defensa y promoción de los derechos de las mujeres en un contexto patriarcal y autoritario, marcado por múltiples violencias. El propósito de Casa E se fundamenta en cuatro componentes: artístico, pedagógico, institucional y comunicativo.
El objetivo del Proyecto VICTUS es promover escenarios de encuentro y participación ciudadana a partir del diálogo entre "interlocutores improbables," que es el término con el que Lederach (2018) define a aquellas personas pertenecientes a grupos que hasta el momento no se reconocían como semejantes ni se escuchaban con respeto porque se consideraban enemigos. Para mí es especialmente importante hablar de este proyecto, no solamente porque hago parte del mismo (en la medida en que participo como "actriz natural" a través del "acto de testimoniar" para la construcción colectiva de un relato incluyente e integrador de las diferentes verdades relacionadas con el conflicto), sino porque actualmente la gran preocupación de todos los que le estamos apostando a la construcción de la paz en Colombia desde diferentes lugares, es encontrar caminos que nos permitan articular las iniciativas - políticas, culturales, simbólicas, académicas, pedagógicas, educativas - orientadas a contrarrestar la cultura del odio y la intolerancia. Ésta diariamente se manifiesta a través de las amenazas y los asesinatos selectivos contra lideresas, líderes sociales y excombatientes de las FARC. En este sentido, cabe recordar que desde la firma de los Acuerdos de Paz hasta la semana pasada (24 de mayo de 2019) han sido asesinados 702 líderes sociales y 135 excombatientes de las FARC (https://www.msn.com/...líderes...excombatientes....sido-asesinados...firma-del-acuerdo/).

Hablando de mi historia como participante del Proyecto VICTUS, tengo que decir que, a principios del año 2016 fui invitada a participar en calidad de asesora externa como integrante de un equipo de apoyo psicosocial promovido por la OIM, donde también participaban profesionales de la Unidad Nacional para las Víctimas y de la Agencia Colombiana de Reintegración. El objetivo de este equipo profesional era el de acompañar durante la primera etapa el complejo proceso de encuentro y aproximación entre ex-combatientes de los diversos grupos armados y víctimas civiles, afectadas por las acciones violentas de dichos grupos, con el fin de contribuir a buscar caminos para la reconciliación a través de una propuesta artística inédita en Colombia, liderada por Casa E.

Debido a que desde hace más de veinte años he venido trabajando en procesos de apoyo y acompañamiento psicosocial a víctimas de la violencia sociopolítica, utilizando el arte como lenguaje de creación y comunicación alternativa, realmente me pareció fascinante trabajar en este Proyecto tan original y novedoso. Como profesional de las ciencias sociales, y desde el ámbito de la psicología social, siempre he sabido que para lograr la paz en Colombia y en cualquier contexto marcado por la deshumanización y la polarización extrema, es totalmente necesario abordar los factores subjetivos de la violencia (emocionales, comportamentales, cognitivos, actitudinales) en la medida en que dichos factores contribuyen a la agudización y la degradación del conflicto armado, que obedece a una serie de factores objetivos de carácter estructural, que se desprenden de las dinámicas socio-económicas, 
políticas y culturales que perpetúan la exclusión, la represión, la injusticia y la inequidad.

Por ello, al constatar que dentro del grupo de víctimas civiles pre-seleccionadas por el Equipo de Casa E para el Proyecto VICTUS en ese momento no había representantes del sector de las víctimas de crímenes de Estado, que es la comunidad humana con la que, por razones personales y políticas, más me he relacionado personal y profesionalmente a lo largo de mi vida, decidí que en lugar de trabajar como parte del equipo asesor de apoyo psicosocial, prefería ser parte del grupo de actores naturales con el fin de poder hablar en ese escenario desde un lugar de enunciación que diera cuenta de la difícil experiencia de los defensores de los derechos humanos, los activistas y los líderes sociales históricamente estigmatizados, perseguidos y obligados al desplazamiento interno o al exilio por razones políticas, como fue mi caso.

Después de casi cuatro años de trabajo arduo, agradezco a la vida haberme dado la oportunidad de ser parte del Proyecto VICTUS en tanto éste me permitió resignificar mi historia personal y profesional. A diferencia de la mayoría de las víctimas del conflicto en Colombia, yo no crecí en una familia campesina, carente de recursos y oportunidades, habitante de las zonas rurales en conflicto. No obstante, al igual que la mayoría de mis compatriotas, crecí con el miedo y la desconfianza que se desprenden del hecho de haber nacido en un país que siempre ha estado en guerra. No sobra decir que pertenezco a una generación de colombianos que en su juventud quedó marcada para siempre por el asesinato de tres candidatos a la Presidencia de la República antes de las elecciones de 1990, y por el Genocidio de la Unión Patriótica, en el que una de las miles de personas asesinadas de manera selectiva e intencional por miembros activos del ejército y los grupos paramilitares, fue mi suegro, el Senador Manuel Cepeda Vargas. ${ }^{2}$

Actualmente, puedo afirmar que lo que más aprecio de la experiencia de trabajo en el Proyecto VICTUS es haber reencontrado el camino de la ritualización de la vida cotidiana, a partir de la cual pude hallarme a mí misma en la esencia de los otros, a través de los acercamientos, las miradas, los silencios, las palabras, los abrazos, los desencuentros, los roces, las confrontaciones, los diálogos, las risas, las lágrimas, los besos, y la ternura.

Me siento transformada, $\mathrm{y}$, a decir verdad, un poco trastornada y asustada ante la novedad que implica aceptar que muchas de mis certezas están cambiando de piel. Me reconozco y me conmuevo de una manera distinta cuando me miro en el espejo de una historia compartida que me revela todos los detalles de la trayectoria de dolor y barbarie en la que nos ha situado esta guerra infame, generación tras generación. No me canso de agradecer la oportunidad de haber podido ver la profundidad de las heridas individuales y colectivas que todavía no hemos podido sanar, y espero poder seguir aportando a este proceso de creación colectiva de un país posible, y aprendiendo de mis compañeros de camino, con quienes comparto el sueño de la paz como legado para las generaciones futuras.

Desde esta perspectiva, en mi calidad de investigadora, actora y participante en el Proyecto VICTUS, estoy interesada en conocer las opiniones de las tres personas que han dirigido y orientado este proceso que comenzó a gestarse en el año 2014, cuando Carlos Arturo Velázquez (Coronel del Ejército y Asesor en Memoria Histórica Militar para el sector Defensa) llegó a la sede de Casa E buscando apoyo en Alejandra Borrero, su directora, para crear y producir una obra de teatro basada en historias reales de valor y superación moral de ex combatientes del Ejército y la Policía Nacional que hubieran resultado gravemente afectados, física y emocionalmente, en el contexto del conflicto armado. Alejandra le respondió que aunque la propuesta le parecía interesante, sería más interesante aún si involucraba a los otros protagonistas del conflicto - tanto víctimas civiles, como desmovilizados de los diferentes grupos armados ilegales - con el fin de transformar los imaginarios deshumanizantes que han contribuido a exacerbar los odios y la polarización en Colombia, después de más de cinco décadas de violencia política y social.

De este modo comenzó el equipo interdisciplinario de Casa E Social a organizar el proceso de entrevistas con el fin de seleccionar a cinco personas de cada uno de los cuatro grupos poblacionales directamente involucrados en el conflicto, a saber: víctimas civiles; militares y policías en retiro; ex guerrilleros de las FARC y el ELN y ex paramilitares de las Autodefensas Unidas de Colombia (AUC). Las personas seleccionadas para ser "actores naturales" de la obra fuimos hombres y mujeres entre los 18 y los 61 años, provenientes de las diferentes zonas del país. Constituíamos una muestra representativa de los distintos sectores que se han visto afectados en el contexto de la violencia sociopolítica a nivel nacional y regional. La mayoría tenemos un buen nivel de escolaridad, y algunos tenemos formación profesional. Hay representación del sector indígena, afro-descendiente, LGTBI, de la población campesina y urbana, de las víctimas de la violencia sexual, el reclutamiento forzado, el secuestro, las minas antipersonal, la desaparición forzada, la tortura, el desplazamiento forzado y el exilio por razones políticas.

Desde el segundo semestre del año 2017 hasta la fecha, el proceso de trabajo al interior del Laboratorio VICTUS se ha ido enriqueciendo en la medida en que se ha presentado en diferentes escenarios, dentro y fuera de Bogotá, donde los diálogos con los diferentes tipos de público han inspirado la generación de una nueva ruta de trabajo encaminada a fortalecer los procesos de construcción de la verdad desde una perspectiva transformadora que contribuya a que el país se reconozca en su historia de dolor y resiliencia colectiva. Hoy más que nunca, dadas las particularidades de la actual coyuntura política, donde es clara la división entre los defensores y los enemigos de los Acuerdos de Paz, es necesario continuar desarrollando el Proyecto VICTUS. 


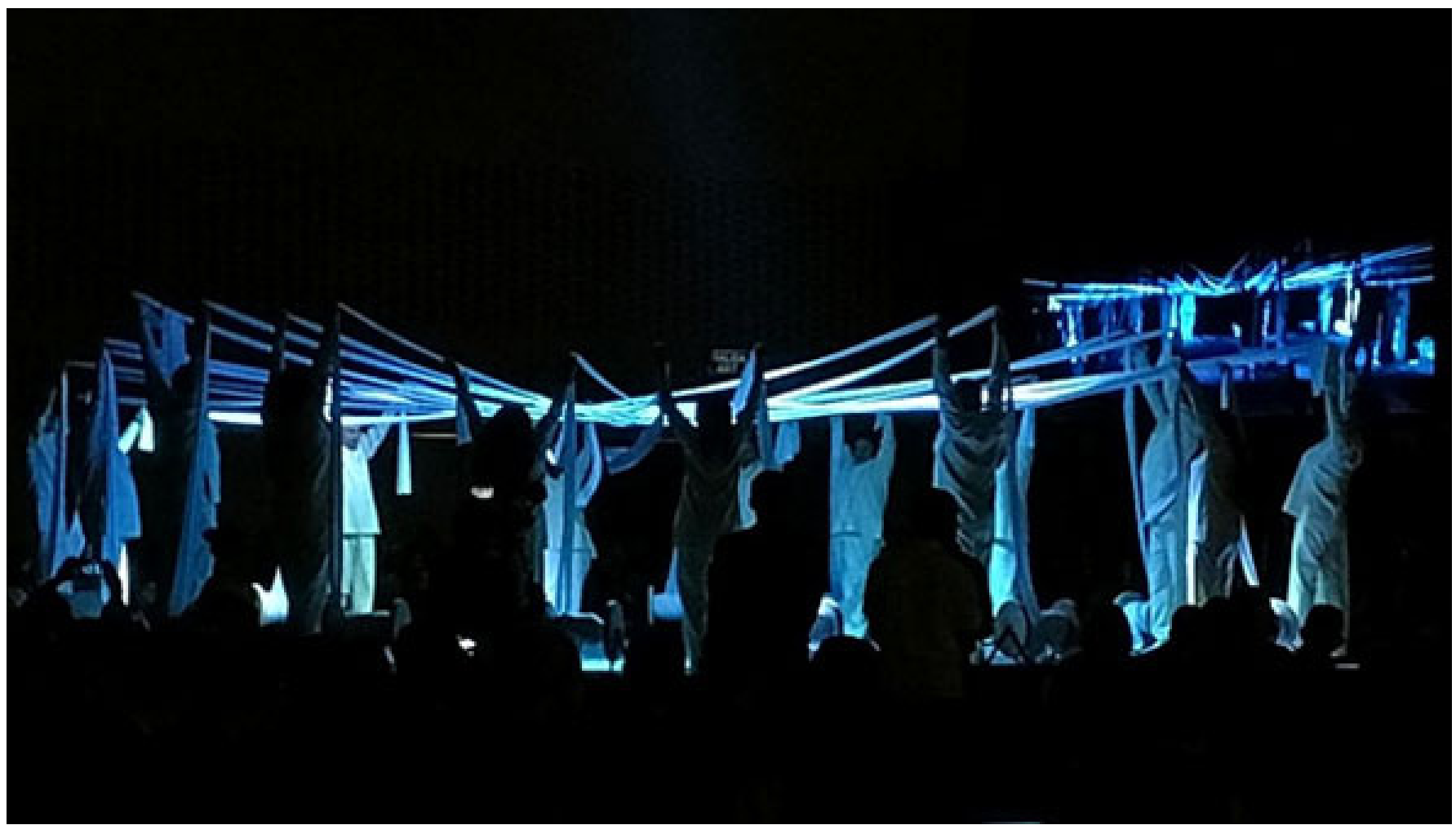

Proyecto VICTUS:

Acto de Inauguración de la 16a Cumbre de Premios Nóbeles de Paz en Bogotá, 2 febrero de 2017

A continuación comparto el diálogo sostenido con las tres personas que coordinan el equipo pedagógico y creativo de Casa E Social en el marco del Proyecto VICTUS. En primer lugar, Alejandra Borrero, que, como dije anteriormente, es la directora del Proyecto VICTUS, y además de actriz, dramaturga es una activista social de gran trayectoria a nivel nacional e internacional, que promueve la defensa de los derechos de las mujeres contra las diferentes manifestaciones de la violencia de género en Colombia. En segundo lugar, María Victoria Estrada (asesora pedagógica y metodológica del proceso) quien estudió psicología infantil, educación pre-escolar y una Licenciatura en educación artística, así como medicina tradicional china, medicina alternativa y bioenergética. En tercer lugar, León David Cobo, el director musical y creador de los paisajes sonoros del Proyecto VICTUS, quien es un músico de carrera por la Pontificia Universidad Javeriana, artista sonoro, docente e investigador. Su trabajo se centra en la recuperación de las lenguas, las historias y la música ancestral de las comunidades indígenas.

Claudia Girón: ¿Para usted cuáles han sido los aprendizajes más significativos a nivel personal y a nivel profesional en el proceso de co-creación del Laboratorio VICTUS, que este año ya comienza su tercera etapa?

Alejandra Borrero: Los aprendizajes más significativos han estado a nivel personal, han sido realmente profundos...Creo que todos los que pasamos por este proceso de
VICTUS, por esta co-creación, cambiamos profundamente... $Y$ no es un una palabra por decirla, realmente ha sido un cambio profundo. Los aprendizajes... Increíblemente, logramos romper paradigmas que cada uno pensaba que no tiene, pero que todos tenemos... De alguna manera, todos, todos, todos los seres humanos discriminamos, y esto fue una manera de romper con ciertas ideas, maneras de pensar... Ciertas ideas que seguían taladrando, digamos, el SER... El SER que, de todas maneras, ya toma otra consciencia. Creo que ese es uno de los aprendizajes más importantes. El otro, la capacidad de ser maleables, de poder pararnos en los pies de los demás, de poder pararnos en los zapatos de los demás, y poder entender desde el lugar del otro, el conflicto, el dolor, la rabia que tiene, el problema que quiere solucionar... De los aprendizajes más significativos... Bueno, esto es algo que ya sabía, y es que cada ser humano es profundamente sabio, así que el aprender de cada uno de ellos ha sido realmente fundamental para tener un pensamiento más plural, más incluyente... Como co-creadora de este proceso y de este proyecto, la humildad también ha sido un aprendizaje increíble; poder permitir que aflore la creatividad y la imaginación.

María Victoria Estrada: VICTUS desde su origen ha contado con una propuesta pedagógica, artística y metodológica que ha constituido un lugar de movilización de pensamiento, y hoy puedo decir que de construcción de conocimiento, desde lo personal, lo profesional lo político y lo social. Contar con una estructura abierta, amplia y flexible, que desde el inicio ofreció un proceso pedagógico en donde 
se consagró un espacio para la participación genuina; no sólo como derecho, sino desde el sentido de lo humano, donde lo espiritual — entendido desde la "expansión de la consciencia que tiene en cuenta al otro, a la otra, a los otros, las otras y lo otro; donde habita el tejido social que nos contiene"ha implicado hasta el momento una propuesta pedagógica y artística que ha sido de ida y vuelta; es decir, que el aprendizaje ha sido para ambas partes, desde el inicio, a partir del momento del encuentro y el reconocimiento, hasta el momento de la evolución y la creación, se mantuvo y se mantiene la fluidez... La primera fase del proyecto fue del 1 de febrero hasta el 26 de mayo del 2016.

Como asesora pedagógica de VICTUS y desde la experiencia del trabajo artístico y creativo, adelantado desde hace un poco más de cuatro décadas, considero que VICTUS como laboratorio ha sido un espacio para romper con prejuicios, con realidades acomodadas, acordadas, ajustadas o admitidas... Es meterse en las entrañas de la guerra y entender que en este país, de una u otra manera, todos somos víctimas del conflicto armado.

León David Cobo: Los aprendizajes han sido muchos. El aprendizaje, digamos, a largo plazo, entender que las artes efectivamente son un camino; que las artes y el arte, efectivamente, es en sí mismo; el arte por sí mismo, por su propia naturaleza... No el arte en relación con la política, el arte en relación con lo psicosocial... ¡NO!... El arte "ÉL," en su propia esencia, en su propia esencia del deseo, del deseo de la creación, del deseo a participar, de la necesidad de decir algo... "ÉL," en su propia esencia, funciona, en términos de los procesos de reconciliación, de los procesos en la construcción de paz. El arte NO es un medio -eso es una cosa muy importante- El arte es EL CAMINO ¿Sí? ... Hay una diferencia $¿$ No?... El arte es el camino a través del cual se recorre, se vive, se hacen prácticas, se reflexiona, se piensa, pero es el arte el camino. Digamos, ha sido muy significativo constatarlo una vez más... El tema del proceso de co-creación, en lo profesional, pues me parece que VICTUS tiene un sonido propio; ha sido una búsqueda muy rigurosa por encontrar cuál es el sonido de VICTUS, cuál es el universo sonoro de VICTUS, el universo musical de VICTUS; cómo se mueven las moléculas en el aire a través de las voces, de las gargantas de los VICTUS, y cómo eso ha generado un discurso, una narrativa propia ¿No?... Ese logro que tiene VICTUS es que VICTUS suena a VICTUS, y a nada más... Él tiene su propio sonido, que nace de su propia voz, de su propia entraña... ¿Por qué nace de su propia voz? Porque hemos tenido en cuenta, en el marco del proyecto pedagógico, reconocer al otro como un ser que tiene información, que tiene memoria y que tiene una cultura. Un ser que se reconoce con esos elementos, ya él es un ser completo, ya trae todo eso. Lo que hemos hecho a partir de las narrativas sonoras es reconocer que los VICTUS son portadores de memoria, que los VICTUS son portadores de información, que los VICTUS son portadores de un constructo cultural que cada uno trae muy distinto, y reconocer que desde esos constructos elaboramos nuestra propia identidad ¿No?... Musical, sonora, en términos de una construcción colectiva, pues esto es una cosa que hacemos entre todos. Entonces, en lo personal, eso, en relación con el arte...Ya lo mencioné antes: el arte es el camino, y se puede constatar a través de VICTUS. El arte por el arte, por el arte. Y lo segundo, eso del sonido propio ¿No?... Tal vez para agregar que Colombia es un país muy complejo donde siempre he tenido mucha curiosidad por entender por qué es que pasó todo esto que pasó, y VICTUS me ha ayudado a entender muchas cosas, pero también a hacerme otras preguntas, $\mathrm{y}$ valoro mucho las otras preguntas porque es desde ahí que se construye.

\section{Claudia Girón: ¿Cuál considera que es el factor prin- cipal para que, a pesar de los conflictos internos y exter- nos, el Proyecto VICTUS se haya mantenido vigente como propuesta pedagógica orientada a mantener la esperanza en un futuro diferente, donde sea posible la reconciliación y la convivencia pacífica entre los colombianos?}

Alejandra Borrero: Yo creo que lo que ha pegado y ha mantenido, digamos "el almizcle" con el que se ha pegado VICTUS, es el amor... Y en eso estoy convencida, el amor que le hemos puesto al Proyecto, el amor que cada uno de nosotros ha puesto dentro del trabajo. Eso como un ingrediente fundamental... Pero también, el considerar que la paz nunca puede ser perfecta; que los seres humanos no somos perfectos, y considerar que cada uno de los que trabajan en VICTUS traen una historia y un bagaje que hace que cada uno tenga sus singularidades, y es por eso que éste es un Proyecto que tiene siempre que estar pendiente de cada uno de ellos... No podemos pretender ni esperar que todos sean perfectos y que todos lleguen al mismo lugar... Cada uno ha tenido su camino, cada uno ha llegado a diferentes lugares, pero todos han llegado a la reconciliación. La convivencia es posiblemente lo más difícil; la convivencia pacífica entre los colombianos... VICTUS es una muestra clara de lo difícil que es la convivencia pacífica entre los colombianos; pero seguimos intentándolo, y seguimos trabajando en esos temas... Creo que VICTUS - y esto también es parte de lo que considero que ha hecho VICTUS se mantenga - es que es una propuesta que sigue creciendo, a pesar de las dificultades económicas; a pesar de no ser un organismo que puede contar con el dinero suficiente para hacer un proceso completo como nos lo hemos propuesto, pues... Y tenemos que parar y esperar a poder conseguir, y mandar otro proyecto, conseguir los dineros... A pesar de todo eso, es una propuesta que crece, y ellos han ido creciendo profundamente dentro del montaje, dentro de la propuesta, y son ya muy capaces de proponer y de ver sus vidas desde otros lugares.

María Victoria Estrada: VICTUS ha sido desde el primer momento "un elogio a la dificultad", tal y como lo plantea Estanislao Zuleta. Esto no ha sido de ninguna manera un 
"mar de mermelada"; ha sido como la vida misma, justamente, contar con un universo pedagógico, metodológico al servicio del arte, implica tener propuesta, pensar, trabajar en equipo, sistematizar para tener en cuenta siempre las habilidades, capacidades, potencialidades y talentos del grupo. En el laboratorio artístico, se conjugan el arte dramático, la música, el arte sonoro, la literatura, las artes plásticas y visuales, el performance... Es allí donde la creación lleva consigo el rompimiento, el quiebre, la evolución, la disolución de los prejuicios anclados en la guerra, en la lucha, en el combate... Es allí, en la creación, donde se genera un estado de ampliación de la consciencia, que ha permitido, a lo largo del proceso, reconocer a los otros y a las otras como legítimos, como iguales en la convivencia, sin discriminación, ni abuso, como lo plantea Humberto Maturana. Se trata justamente de comprender qué es lo que este país ha vivido a lo largo de más de 70 años, si partimos del año de 1.948, y, desde esa comprensión, entender "que detrás de una verdad, hay múltiples verdades," tal y como lo plantea Cristina, una de las integrantes del grupo.

Encontrar en VICTUS un lugar para respirar conscientemente, para meditar y ofrendar al universo los cuatro elementos en cada uno de los ensayos y las presentaciones; encontrar un territorio para conversar-por más difíciles que sean las circunstancias por las que se esté atravesando-un espacio para la confianza, el respeto, el AMOR, con mayúsculas, es realmente un homenaje a la memoria, la reconciliación, la convivencia y las múltiples verdades que habitan en VICTUS.

León David Cobo: El factor principal para mí, pues no es solo un factor, es un sistema de factores. Voy a hablar de los que yo considero que son importantes. Uno es la escucha, la escucha del otro, reconocer al otro como un igual en la convivencia, la escucha del otro en esa precariedad, en esa dificultad que plantea la diferencia. Escuchar al otro, pero al otro con el que no estoy de acuerdo, me obliga a mí a ponerme en un lugar elevado; elevado para yo poder tener la posibilidad consciente, amorosa y real de escucharlo. Entonces la escucha como un factor importante... Sobre eso yo he escrito mucho... El otro tema importante aquí, tiene que ver con una posibilidad de creación... Nos conecta la creación.. Estamos creando, estamos haciendo arte, no estamos haciendo otra cosa; es arte lo que estamos haciendo cada vez que nos paramos en un escenario, cada vez que improvisamos, que reflexionamos, que leemos, estamos haciendo arte. VICTUS como propuesta creativa es el otro factor que yo creo que es importante en relación con las prácticas artísticas. Y yo diría que un tercer factor es el espacio que brinda Casa E; es un espacio de aprendizaje, un espacio en el que las instituciones están aprendiendo a hacer eso que es la reconciliación, que se hace a través del arte. Yo valoro ese espacio, ese espacio en el que nos podemos encontrar, lo valoro como primer factor. Está la escucha, está el arte, la creación, y está el espacio que propicia el encuentro, que propicia no solamente la creación, sino desde la propuesta conceptual, la propuesta pedagógica de Casa E, la propuesta pedagógica, artística y metodológica "al servicio de"... Yo valoro la propuesta pedagógica como factor principal, valoro el arte como valor principal, y la escucha, como ya lo dije anteriormente.

\section{Claudia Girón: ¿Cuáles han sido los principales retos y desafios a los que usted se ha visto enfrentado(a), teniendo en cuenta, tanto la diversidad de versiones de la historia del conflicto-encarnadas por los actores naturales que participan en la obra-como la complejidad del contexto de transición hacia la paz en un país tan dividido por la desconfianza y la polarización política y social?}

Alejandra Borrero: Los principales retos han sido mantener el grupo. Yo creo que, justamente, y no por la diversidad de versiones de la historia del conflicto, hemos aprendido a ser flexibles, a escuchar y, posiblemente, uno de los mayores aprendizajes que deberíamos tener como colombianos serían estos cinco años de diálogo [Refiriéndose a los diálogos entre el Gobierno de Juan Manuel Santos y la Guerrilla de las FARC en La Habana, que comenzaron en el año 2012)]... Creo que el diálogo es la manera de salir del meollo, y de meternos en el rollo que corresponde. En VICTUS nuestros diálogos han sido tan profundos, que muchas veces sentimos que son diálogos que todavía el país no está listo para escuchar. La diversidad de versiones de las historias del conflicto forman parte de esa manera de pensar incluyente, y donde todos, realmente todos, aprendimos que todos tienen derecho, $\mathrm{y}$ que todos ha sido víctimas, de una manera u otra. Pero con respecto al contexto de transición hacia la paz de este país, la polarización y la desconfianza, pues eso genera una gran desconfianza en ellos también ¿no? en todos nosotros, los colombianos... La paz se ha convertido casi en una palabra proscrita, y estamos muy asustados todos los que estamos trabajando por la paz, porque la situación es realmente precaria, y es realmente precaria también para ellos; así que muchos de ellos sufren enfermedades; muchos de ellos están en situaciones muy complejas económicamente, y realmente no estamos viendo soluciones, así que la situación es muy precaria... VICTUS, de alguna manera, sobrevive por un hilo invisible que lo sostiene, y yo creo que es el amor.

María Victoria Estrada: Los retos y los desafíos han estado fundamentalmente anclados en creer para proponer y sentir para realizar... En mantener el espíritu creador, mantener en el horizonte de sentido al ser humano habitado de las cualidades trabajadas en VICTUS a lo largo de estos cuatro años. A lo largo de estos cuatro años, donde el arte ha sido desde su dimensión creadora lo que ha generado los procesos de transformación que se han revelado, tanto al interior del grupo, como hacia el público; un público que siempre, en todas las funciones realizadas, además de pararse a aplaudir como un homenaje a VICTUS, ha desentrañado desde la obra misma los referentes históricos y culturales que han hecho de la guerra nuestra cotidianidad... Un público que ha 
reconocido las verdades impuestas a lo largo de los años y ha sido capaz de llorar públicamente, reconociendo el aporte de este trabajo a la comprensión del conflicto armado que hemos vivido como colombianas y colombianos. Otro reto, desafío o incertidumbre frente al Proyecto VICTUS lo constituye la situación por la que hoy atraviesa el país... Esa transición a la PAZ, con mayúsculas, tan obstaculizada, tan habitada por ideologías, y con ella, la polarización que no les permite a las colombianas y colombianos comprender la dimensión ética del cuidado, que nos exige hoy el momento histórico de la transición que vivimos. La hostilidad que vivimos está alimentada ideológicamente por los principios y criterios de la guerra, la competencia, las armas.. Como si la guerra fuera lo fundamental.

El desafío que afrontamos en VICTUS es muy grande: Se trata de continuar generando y contribuyendo con el público al que VICTUS puede llegar y se conecta con la obra como hasta ahora ha sucedido, aportando otra mirada desde el trabajo pedagógico y artístico realizado, que ofrece un camino para esclarecer las verdades que excombatientes de los diferentes grupos armados ilegales, militares retirados de la policía y el ejército y víctimas de la sociedad civil presentan desde sus propias historia de vida.

León David Cobo: Los principales retos y desafíos yo los puedo enumerar en términos de varias categorías. Una categoría es la de las relaciones humanas. Creo que VICTUS es, como todos los procesos donde hay seres humanos, desde un circo, hasta pasar por una oficina, o una liga de fútbol; es decir: donde se reúnen seres humanos, siempre hay el reto de resolver el conflicto, en todos estos grupos que he mencionado...Cuando se reúne la Asociación de Cafeteros, o las Sociedades de las Cámaras de Comercio... En este caso, VICTUS también es un grupo de seres humanos que plantea unos desafíos particulares ¿No? es decir, la diferencia, la diversidad, el ver al otro como un igual en la convivencia implica también verse uno, verse uno con el otro, ponerse uno al mismo nivel del otro. Maturana dice "que uno no se puede poner en los zapatos del otro", pero yo sí creo que uno puede acompañar al otro, y ese ha sido el desafío de VICTUS... Siento que se ha trabajado eso desde el amor... El amor ha sido muy importante en ese reto, en ese desafío de mantener a un grupo unido, llámese banda de rock, equipo de rugby, hasta un Ministerio completo de Cultura... Digamos, un reto y un desafío es mantener a todo un equipo vinculado en una sola causa. Tratándose de personajes tan disímiles, ha sido todo un reto, y uno de los aprendizajes, como ya mencioné, ha sido la escucha. El otro reto que también tiene que ver con VICTUS en relación con el espacio-tiempo del país, es que en los últimos años ha cambiado mucho: desde un espíritu ferviente en unos y amargo en otros en relación con la paz, la firma de la paz, y lo que pasa ahora, pues, con el cambio de gobierno, que tiene otras actitudes en relación con esa paz, y que tiene otra manera de pensarse la paz. Entonces me parece que VICTUS siempre va a ser un grupo vigente, porque yo no veo en el corto plazo que el país vaya a cambiar; al contrario...Entonces creo que VICTUS, pues, será vigente, y ese será uno de los retos y los desafíos: ver de qué manera se va a mantener vigente y necesario este proyecto.

\section{Claudia Girón: ¿Cómo cree usted que el Proyecto VICTUS ha aportado y puede seguir aportando a construir caminos hacia la reconciliación, la convivencia y la paz imperfecta a partir de la elaboración de un relato incluyente de las dife- rentes verdades relacionadas con los orígenes, dinámicas $e$ impactos del conflicto en Colombia?}

Alejandra Borrero: Primero que todo, hay que decir que el conflicto no ha terminado en Colombia, y que muchas de las dinámicas de la guerra han vuelto, así que éste sigue siendo todo un proceso dinámico que sigue construyéndose a pesar de, digamos, a pesar del impacto que ha tenido el conflicto en Colombia... ¿Cómo puede seguir aportando caminos hacia la reconciliación, la convivencia y la paz? Mostrando lo imposible...VICTUS realmente es capaz de mostrar que, a pesar de todo, ahí estamos...Ahí estamos todos parados desde hace tres años, y más allá de mostrar a los seres humanos que están detrás de todo este dolor; seres que están golpeados, y que también están luchando día a día por sobrevivir... VICTUS no solamente ha aportado con la visión que el público tiene al ver que lo imposible es posible sobre el escenario, sino yo creo que ha aportado en cada uno de ellos para una mejor vida, para una mejor situación de vida de cada uno de ellos. Por supuesto no económica, pero sí de paz interior, de trabajar digamos hacia cada uno de nosotros esta paz, que es la verdadera paz que necesitamos. Y son precisamente estas miradas incluyentes las que permitirán y permiten hacer una lectura diferente del entorno. Así que creo que ese es uno de los grandes aportes de VICTUS.

María Victoria Estrada: En el recorrido por las preguntas que esta entrevista ha ofrecido, siento que esta pregunta ya ha sido respondida. Solo agregaría que la reconciliación, la convivencia y la paz implican que como seres humanos, como hombres y como mujeres, ciudadanos y ciudadanas de este país y del globo terráqueo, necesitamos entender de manera trascendente lo que significan estos conceptos de reconciliación, convivencia y paz interiormente, para que los podamos entender afuera... Necesitamos pausa, necesitamos silencio $y$ un alto en el camino, estamos habitados por demasiado ruido, traducido en mentiras que se van volviendo verdades, en un contexto donde se han naturalizado tanto el conflicto armado, como la violencia, en la medida que nos las repiten cada día. Para cerrar les dejo con este epígrafe de W. Goethe, que nos ha acompañado en estos cuatro años de existencia de VICTUS; epígrafe que nos deja un haz de luz para comprender que es un proceso que al menos ya comenzó: "No hay que dar pasos que conduzcan a la meta; es menester que cada paso sea una meta, sin dejar de ser un paso." 
León David Cobo: Bueno, VICTUS ha aportado mucho, pero también, sin lugar a dudas, puede seguir aportando...A través de VICTUS solamente se ha visto la punta del iceberg, y no hemos logrado todavía...Y eso es lo bonito del proyecto: que tiene mucho camino por recorrer en varios sentidos... En el primer sentido, VICTUS tiene que, definitivamente, darse a conocer de manera más masiva. VICTUS tiene que presentarse más en otras ciudades; VICTUS tiene que hacer giras internacionales; giras por Latinoamérica, donde tiene mucho sentido en términos de la reflexión que se pueda dar en países Latinoamericanos que son similares al nuestro, por las mismas problemáticas; pero también en países Europeos, donde Latinoamérica se vea a través de VICTUS...Colombia puede contarle al mundo que sí se puede reconciliar, que hay otras maneras de hacer las cosas en Colombia. Ojalá podamos ir a Estados Unidos, ojalá podamos ir al África...Es decir, desde lo que significa la circulación del proyecto que ya tenemos, siento que hay que hacer mucho más. $Y$ en términos de nuevos materiales, siempre lo he dicho, es el momento de hacernos nuevas preguntas; es el momento de seguir problematizando nuestro país... El país que tenemos ahora no es el mismo de hace tres años, cuando hicimos VICTUS, y vale la pena, ya habiendo hecho "la reconciliación, la memoria," pues... hacer un nuevo tema que nos convoque a todos ahora, desde las diferencias, desde lo que siempre hemos sido, y que nos plantée los nuevos derroteros del país presente.

\section{Obras Citadas}

Alto Comisionado para la Paz: http://www.altocomisionadoparalapaz.gov.co/procesos-y-conversaciones/proceso-de-paz-conlas-farc-ep/Paginas/PR-Sistema-integral-de-Verdad-Justicia-Reparacion-y-no-Repeticion.aspx

CASTILLEJO, Alejandro (2018). La paz desde lo pequeño. https:/lapipa.co/la-paz-desde-lo-pequeno-alejandro-castillejo/

Comisión de la Verdad, 06 de 2018: https://comisiondelaverdad.co/actualidad/noticias/los-dialogos-improbables-de-john-paullederach-una-forma-de-construir-confianza-en-los-territorios

FEIERSTEIN, Daniel. 2007. El genocidio como práctica social. Entre el nazismo y la experiencia argentina. Buenos Aires: Fondo Cultura Económica.

\section{Notas}

1. Según Daniel Feierstein (2007), la construcción de un "enemigo interno" a quien es legítimo aniquilar se basa en la configuración socio-histórica, política, jurídica y cultural de "una otredad negativa," que consiste en generar un proceso de "deconstrucción" de un grupo humano catalogándolo como "anormal" (inhumano," nocivo, peligroso, contagioso) en la medida en que atenta contra una mayoría normalizada.

2. Manuel Cepeda Vargas, Senador de la República por el partido político Unión Patriótica (UP) fue asesinado por miembros del Ejército Nacional y de los grupos paramilitares el 9 de agosto de 1994. Era el padre de Iván Cepeda Castro, con quien estuve casada durante quince años. Juntos, a raíz de nuestro trabajo de denuncia a nivel nacional e internacional, logramos establecer las responsabilidades del Estado colombiano, tanto por acción, como por omisión, en el asesinato de Manuel Cepeda Vargas, demostrando que no se trató de un caso aislado, en la medida en que fue cometido en el marco de una serie de acciones sistemáticas de exterminio contra los líderes y miembros de la UP. A raíz de que las denuncias realizadas lograron aportar elementos para el esclarecimiento de la verdad en el caso del Genocidio contra la Unión Patriótica-cuyo saldo, según los últimos informes de la Corporación Reiniciar y del Centro Nacional de Memoria Histórica, es de más de 6.000 víctimas - fuimos amenazados de muerte en el año de 1999 y obligados a exiliarnos en Francia hasta el año 2004. Mi relato en el Proyecto VICTUS sobre mi experiencia como sobreviviente y persona retornada del exilio por razones políticas, se teje con los relatos de los demás participantes en una trama narrativa que resalta la importancia de reconocernos como semejantes en la vulnerabilidad y en la dignidad humana. 